\title{
DETERMINAÇÃo RÁPIDA E SIMULTÂNEA DE PROPIFENAZONA, PARACETAMOL E CAFEÍNA UTILIZANDO ANÁLISE POR INJEÇÃO EM BATELADA COM DETECÇÃO AMPEROMÉTRICA
}

\author{
Weberson Pereira Silva, Luiz André Juvêncio Silva, Rodrigo Alejandro Abarza Muñoz e Eduardo Mathias Richter* \\ Instituto de Química, Universidade Federal de Uberlândia, Av. João Naves de Ávila, 2121 - 38400-902 Uberlândia - MG, Brasil
}

Recebido em 17/05/2017; aceito em 29/08/2017; publicado na web em 16/10/2017

\begin{abstract}
FAST SIMULTANEOUS DETERMINATION OF PROPIFENAZONE, PARACETAMOL AND CAFFEINE USING BATCH INJECTION ANALYSIS WITH AMPEROMETRIC DETECTION. A fast and simple procedure for simultaneous determination of propyphenazone (PRO), paracetamol (PAR) and caffeine (CAF) was performed using batch injection analysis with multiple pulse amperometric detection (BIA-MPA). Three potentials pulses were selected for the analysis: +0.90 V/50 ms: only PAR was oxidized; +1.20 V/50 ms: oxidation of PAR and PRO without the interference of CAF; $+1.60 \mathrm{~V} / 50 \mathrm{~ms}$ : oxidation of the three compounds (PAR, $\mathrm{PRO}$ and $\mathrm{CAF})$. The sample aliquot $(150 \mu \mathrm{L})$ was directly injected onto the boron-doped diamond (BDD) electrode immersed in a BIA cell. The analytical characteristics of the proposed method include high analytical frequency (75 injections per hour), good stability (RSD < 3.9\%; $\mathrm{n}=10)$, and low detection limits $\left(0.3,0.2\right.$ and $0.1 \mathrm{mg} \mathrm{L}^{-1}$ for PRO, PAR and CAF, respectively). The proposed method yielded similar results to those obtained by liquid chromatography at a $95 \%$ confidence level.
\end{abstract}

Keywords: boron doped diamond electrode (BDDE); multiple-pulse amperometric detection; portable electrochemical method; pharmaceutical.

\section{INTRODUÇÃO}

A propifenazona (PRO) é um anti-inflamatório não-esteroidal $(\mathrm{AINE})^{1,2}$ do grupo das pirazolonas de rápida absorção indicado como analgésico e antipirético, sendo duas vezes mais potente que a aspirina. ${ }^{3}$ Outro AINE de ação analgésica e antipirética, amplamente utilizado em função de seus reduzidos efeitos colaterais é o paracetamol (PAR). ${ }^{4-6}$ Entretanto, o excesso de PAR pode causar problemas à saúde, como inflamação no pâncreas, erupções cutâneas, doenças hepáticas e hepatotoxicidade fatal. ${ }^{7,8} \mathrm{~A}$ fim de se obter efeito sinérgico, o PAR e PRO são comumente, associados a cafeína (CAF), um estimulante do grupo das xantinas que apresenta ação analgésica secundária. ${ }^{9} 10$

A combinação dos três princípios ativos (PRO, PAR e CAF) gera uma atividade terapêutica mais eficiente devido a atuação de cada droga em sítios ativos diferentes no organismo proporcionando maior eficácia analgésica e maior rapidez no início de ação quando compara-se com as drogas administradas separadamente. ${ }^{11,12}$ Assim, é possível concluir que o uso de formulações farmacêuticas contendo mais de um princípio ativo apresenta vantagens. Dessa forma, no controle de qualidade deste tipo de medicamento, o uso de metodologias analíticas que permitem determinações simultâneas é altamente desejável. Alguns métodos para a determinação simultânea de PAR, PRO e CAF foram localizados na literatura. Os procedimentos empregados anteriormente para este fim foram a cromatografia líquida de alta eficiência, ${ }^{2,10,13,14}$ espectrofotometria UV-Vis, ${ }^{15-18}$ cromatografia capilar eletrocinética micelar, ${ }^{19}$ eletrocromatografia planar pressuri$\mathrm{zada}^{20}{ }^{20}$ cromatografia de camada delgada de alto desempenho ${ }^{20} \mathrm{e}$ voltametria de onda quadrada. ${ }^{21}$

O sistema conhecido como análise por injeção em batelada com detecção amperométrica de múltiplos pulsos (BIA-MPA, do inglês "Batch Injection Analysis with Multiple Pulse Amperometric detection") vem sendo usado com sucesso em determinações simultâneas de dois ${ }^{22,23}$ ou três ${ }^{24,25}$ compostos. Ademais, o método permite ainda a limpeza constante da superfície do eletrodo de trabalho por meio

*e-mail: emrichter@ufu.br do uso de pulso de potencial adicional com este propósito. O sistema BIA-MPA permite a realização de análises simples e rápidas com a injeção de uma pequena alíquota de amostra ou solução padrão (geralmente de 50 a $150 \mu \mathrm{L}$ ) com o auxílio de uma micropipeta (geralmente eletrônica) sobre a superfície do eletrodo de trabalho que está imerso em um grande volume de eletrólito suporte. ${ }^{26,27}$ O sistema BIA acoplado a MPA apresenta diversas características desejáveis, como o uso de instrumentação de baixo custo (aquisição e manutenção), baixo consumo de reagentes e amostras (geração de volumes mínimos de resíduos por análise), elevada frequência analítica e boa estabilidade. ${ }^{22,28}$

Neste trabalho é apresentado um novo método eletroquímico usando a análise por injeção em batelada com detecção por amperometria de múltiplos pulsos para determinação rápida e simultânea de PRO, PAR e CAF. A análise é possível através de injeção de volume reduzido de solução $(150 \mu \mathrm{L})$ diretamente sobre a superfície do eletrodo de trabalho.

\section{PARTE EXPERIMENTAL}

\section{Reagentes, soluções e amostras}

PAR, CAF e ácido acético foram obtidos da Synth (Diadema Brasil), PRO e ácido bórico da Sigma-Aldrich (St. Louis, MO, USA), e ácido sulfúrico, etanol, hidróxido de sódio e metanol da Vetec (Rio de Janeiro, Brasil).

Todas as soluções foram preparadas com água deionizada (Millipore Direct-Q3) com uma resistividade superior a 18,0 $\Omega \mathrm{cm}$. Os reagentes eram de grau analítico e foram utilizados sem purificação prévia. Solução de ácido sulfúrico $\left(0,1 \mathrm{~mol} \mathrm{~L}^{-1}\right)$ foi usada como eletrólito suporte.

As soluções padrão de PAR, PRO e CAF foram preparadas em etanol e diluídas no eletrólito suporte antes de todos os experimentos. As amostras farmacêuticas contendo PAR, PRO e CAF foram obtidas de farmácias locais na forma de comprimidos. Para as análises, quatro comprimidos foram pesados, pulverizados e homogeneizados, e posteriormente algumas porções foram retiradas, pesadas e dissolvidas 
em etanol (solução estoque) e em seguida diluída para uma concentração adequada no eletrólito suporte composto por $\mathrm{H}_{2} \mathrm{SO}_{4} 0,1 \mathrm{~mol} \mathrm{~L}{ }^{-1}$.

\section{Instrumentação}

As medidas eletroquímicas foram realizadas utilizando um potenciostato/galvanostático PGSTAT $128 \mathrm{~N}$ (Metrohm Autolab B.V.) controlado pelo software GPES 4.9.007. Como eletrodo de referência e auxiliar foram utilizados respetivamente um eletrodo miniaturizado de $\mathrm{Ag} / \mathrm{AgCl}$ saturado com $\mathrm{KCl}^{29}$ e um fio de platina. Um eletrodo de BDD composto por uma placa a base de silício $(0,7$ x $0,7 \mathrm{~cm}$ ) com espessura de aproximadamente $1,2 \mu \mathrm{m}$ com nível de dopagem de boro de 8000 ppm foi utilizado como eletrodo de trabalho. Antes de usar o eletrodo BDD pela primeira vez (nova peça de BDD), o material foi pré-tratado eletroquimicamente aplicando $+0,01$ A por $1000 \mathrm{~s}$ em solução tampão Britton-Robinson $0,12 \mathrm{~mol} \mathrm{~L}^{-1}$ (pré-tratamento anódico). Em um segundo passo, aplicou-se -0,01 A durante $1000 \mathrm{~s}$ numa solução de $0,1 \mathrm{~mol} \mathrm{~L}^{-1}$ de $\mathrm{H}_{2} \mathrm{SO}_{4}$ (pré-tratamento catódico). Após o primeiro pré-tratamento, o eletrodo de BDD foi pré-tratado apenas catodicamente uma vez no início do dia de trabalho. Se o eletrodo não for utilizado durante alguns dias, ambos os pré-tratamentos (anódicos e catódicos) são novamente necessários. Este pré-tratamento é semelhante ao utilizado em trabalhos previamente publicados. ${ }^{30,31}$

A célula BIA utilizada nos experimentos foi construída na oficina mecânica da Faculdade de Engenharia Mecânica da Universidade Federal de Uberlândia, como descrita em estudos anteriores. ${ }^{32}$ Nesta célula, a área do eletrodo é definida utilizando um O-ring de borracha com $0,5 \mathrm{~cm}$ de diâmetro (área do eletrodo $=0,2 \mathrm{~cm}^{2}$ ). Um eletrodo de $\operatorname{BDD}(0,7 \times$ x $0,7 \mathrm{~cm})$ foi pressionado contra o O-ring (no fundo da célula BIA) com a ajuda de parafusos e uma base metálica (placa de cobre) sendo o contato elétrico com o eletrodo de trabalho conseguido através da base metálica. O pré-tratamento eletroquímico do eletrodo BDD é realizado com o eletrodo posicionado na célula BIA. Todos os experimentos foram realizados com a solução no interior da célula BIA sob agitação mediante a utilização de um micromotor DC ligado a uma haste mecânica em polipropileno imersa na solução, conforme já descrito anteriormente. ${ }^{33} \mathrm{~A}$ velocidade de agitação foi controlada através de uma fonte universal de tensão variável de 3 a 12 V. Os experimentos foram realizados com a velocidade de agitação de $1400 \mathrm{rpm}$ (aplicação de 5 V). A injeção das soluções na célula BIA foi realizada com uma micropipeta eletrônica motorizada (Eppendorf Multipette ${ }^{\circledR}$ Stream) com uma distância constante entre a superfície do eletrodo de trabalho e o Multipette ${ }^{\circledR}$ Combitip $₫(\approx 2 \mathrm{~mm})$ durante a injeção. ${ }^{34}$ Maiores detalhes sobre células BIA (arte de preparo e modo de utilização) podem ser obtidos em artigos publicados anteriormente ${ }^{28,32,35-37}$ e em vídeos ${ }^{38,39}$ disponibilizados na internet.

Os resultados obtidos por BIA-MPA na análise de amostras farmacêuticas contendo PAR, PRO e CAF foram comparados com os obtidos por HPLC. ${ }^{15}$ Nestas análises foi utilizado o equipamento Shimadzu LC-10 VP com detector UV-Vis (SPD - 10AV), coluna cromatográfica com fase estacionária C18 (Phenomenex, $250 \mathrm{~mm}$ x 4,60 mm, $5 \mu \mathrm{m}$ ), bomba de duplo pistão LC-10AD-VP e injetor manual com alça de amostragem de $20 \mu \mathrm{L}$. A fase móvel era composta por metanol e água na proporção 55:45 (v/v). O comprimento de onda fixado para a determinação dos três compostos foi de 260 nm e a vazão da fase móvel foi de $1,0 \mathrm{~mL} \mathrm{~min}^{-1}$.

\section{RESULTADOS E DISCUSSÃO}

Um trabalho publicado recentemente contém algumas informações importantes sobre os três compostos em estudo no presente trabalho (PAR, PRO e CAF). ${ }^{21}$ As três moléculas, presentes na mesma amostra farmacêutica, apresentam potenciais de oxidação relativamente constantes e separados $(+0,80 \mathrm{~V},+1,30 \mathrm{~V}$ e $+1,55 \mathrm{~V}$ para PAR, $\mathrm{PRO}$ e CAF, respectivamente) usando $\mathrm{H}_{2} \mathrm{SO}_{4} 0,1 \mathrm{~mol} \mathrm{~L}{ }^{-1}$ como eletrólito suporte e BDD como eletrodo de trabalho. Estes estudos foram realizados por voltametria cíclica (sistema estacionário). Com o intuito de obter um método analítico mais rápido, preciso e sensível, o comportamento eletroquímico dos três compostos foi verificado empregando o sistema BIA-MPA. Neste estudo, 10 pulsos de potenciais foram aplicados ao eletrodo de BDD $(+0,60,+0,70$, $+0,80,+0,90,+1,00,+1,20,+1,30,+1,40,+1,60$ e $+1,80 \mathrm{~V} ; 70 \mathrm{~ms}$ cada) repetidamente em função do tempo e 10 amperogramas distintos foram registrados no mesmo experimento. Soluções contendo $10 \mathrm{mg} \mathrm{L}^{-1}$ de CAF, $30 \mathrm{mg} \mathrm{L}^{-1}$ de PRO ou $50 \mathrm{mg} \mathrm{L}^{-1}$ de PAR foram injetadas sequencialmente e a partir dos valores de corrente detectados em cada pulso de potencial (amperogramas), os voltamogramas hidrodinâmicos mostrados na Figura 1 foram construídos.

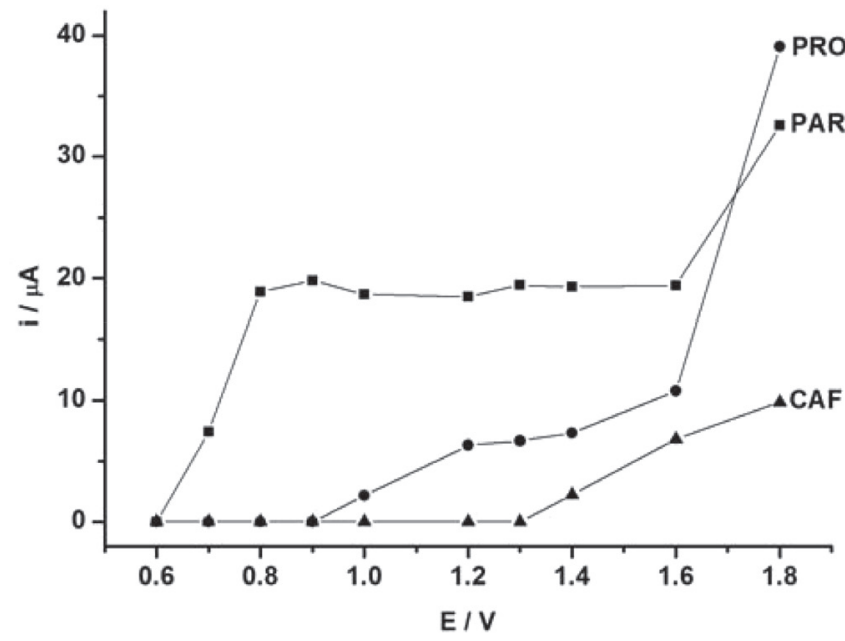

Figura 1. Voltamogramas hidrodinâmicos obtidos pela injeção de soluções contendo separadamente $10 \mathrm{mg} \mathrm{L}^{-1}$ de CAF (A), $30 \mathrm{mg} \mathrm{L}^{-1}$ de PRO (O) ou $50 \mathrm{mg} \mathrm{L}^{-1}$ de PAR (ם) no sistema BIA-MPA. Pulsos de potenciais aplicados: $+0,60,+0,70,+0,80,+0,90,+1,00,+1,20,+1,30,+1,40,+1,60 e+1,80 \mathrm{~V}$, $70 \mathrm{~ms}$ cada; eletrólito suporte: $\mathrm{H}_{2} \mathrm{SO}_{4} 0,1 \mathrm{~mol} \mathrm{L^{-1 }}$; volume injetado: $150 \mu \mathrm{L}$; velocidade de injeção: $75 \mu \mathrm{L} \mathrm{s}^{-1}$

A partir dos voltamogramas hidrodinâmicos apresentados na Figura 1, pode-se observar que a partir do potencial de $+0,6 \mathrm{~V}$ ocorre a oxidação do PAR, onde ocorre a transferência de dois prótons e dois elétrons. ${ }^{40,41} \mathrm{~A}$ oxidação da PRO se inicia próximo ao potencial de $+1,0 \mathrm{~V}$ com a transferência de um elétron e sem a transferência de prótons. ${ }^{21}$ A oxidação da CAF se inicia em potenciais próximos a $+1,3$ $\mathrm{V}$ com a transferência de 4 prótons e 4 elétrons. ${ }^{5,9,42}$ Os voltamogramas hidrodinâmicos apresentados na Figura 1 também mostram que entre os potenciais de $+0,60 \mathrm{~V}$ e $+0,90 \mathrm{~V}$ ocorre a oxidação do PAR sem a interferência da PRO e CAF. Entre $+0,9 \mathrm{~V}$ e +1,3 V ocorrem a oxidação do PAR e PRO sem a interferência da CAF em potenciais acima de $+1,3 \mathrm{~V}$, os três compostos são oxidados.

A partir destas informações, um esquema de aplicação de pulsos de potenciais foi proposto e otimizado $(+0,9 \mathrm{~V} / 50 \mathrm{~ms} ;+1,2 \mathrm{~V} / 50 \mathrm{~ms}$ e $+1,6 \mathrm{~V} / 50 \mathrm{~ms}$ e $0,0 \mathrm{~V} / 100 \mathrm{~ms}$ ) com o intuito de determinação simultânea dos três compostos por BIA-MPA (Figura 2A). Na Figura 2B são mostrados os amperogramas obtidos na injeção em triplicata de soluções contendo: (i) somente PAR $30 \mathrm{mg} \mathrm{L}^{-1}$; (ii) somente PRO $18 \mathrm{mg} \mathrm{L}^{-1}$; (iii) somente CAF $6 \mathrm{mg} \mathrm{L}^{-1}$; (iv) solução contendo PAR $+\mathrm{PRO}+\mathrm{CAF}\left(30+18+6 \mathrm{mg} \mathrm{L}^{-1}\right.$, respectivamente $)$. Os valores de concentração das soluções de PAR, PRO e CAF foram selecionados em função dos valores de concentração destes compostos tipicamente 

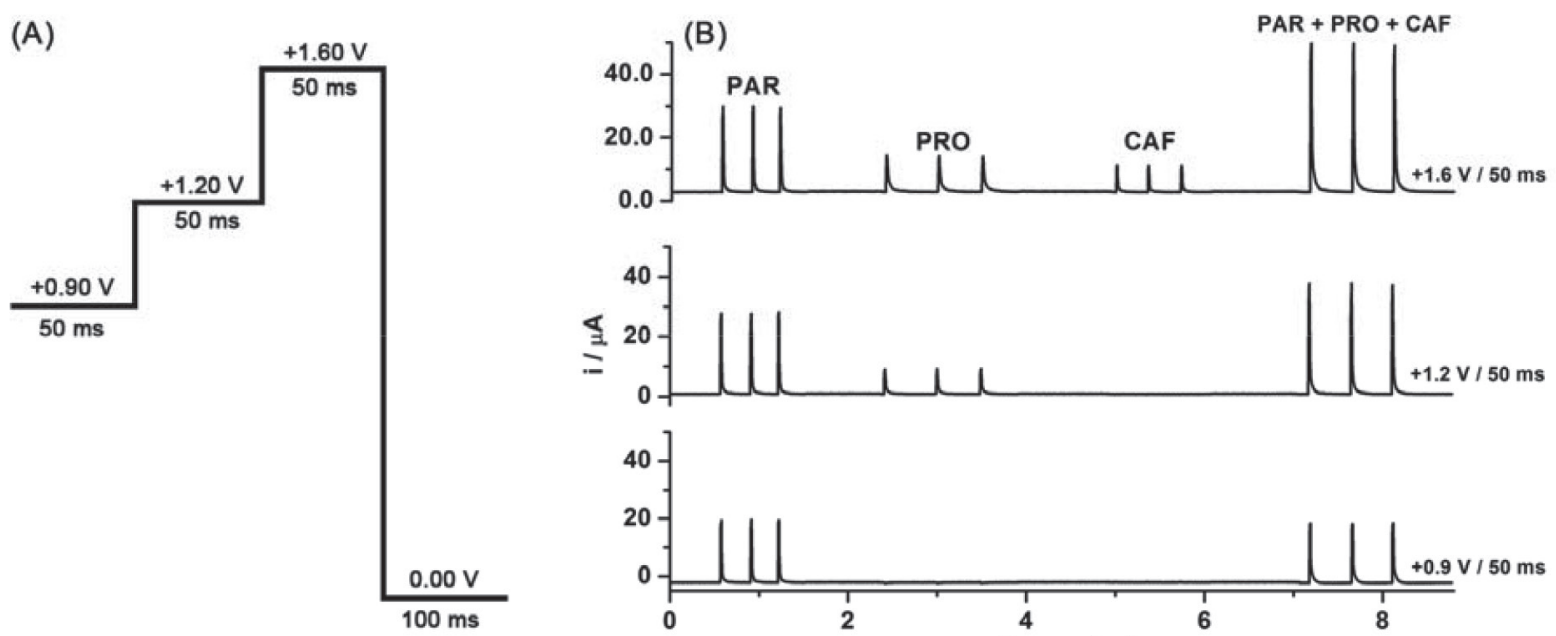

$100 \mathrm{~ms}$

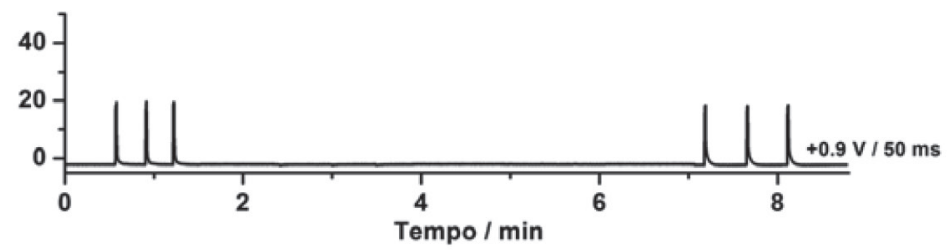

Figura 2. (A) Esquema de aplicação de pulsos de potencial; (B) Amperogramas obtidos após injeções de soluções contendo somente PAR (30 mg $\left.L^{-1}\right)$, somente PRO (18 mg L L $\left.\mathrm{L}^{-1}\right)$, somente CAF $\left(6 \mathrm{mg} \mathrm{L}^{-1}\right)$ e PAR + PRO +CAF $\left(30+18+6 \mathrm{mg} \mathrm{L}^{-1}\right.$, respectivamente). Velocidade de agitação da solução no interior da célula: $1400 \mathrm{rpm}$; volume de injeção: $150 \mu \mathrm{L}$; velocidade de injeção: $100 \mu \mathrm{L} \mathrm{s} \mathrm{s}^{-1}$

encontrados em amostras farmacêuticas (250 mg de PAR, $150 \mathrm{mg}$ de PRO e $50 \mathrm{mg}$ de CAF).

A partir da Figura 2 é possível observar que o PAR é oxidado no potencial de $+0,90 \mathrm{~V}$ sem a interferência da $\mathrm{PRO}$ e CAF, pois respostas similares foram obtidas neste potencial na presença ou na ausência dos demais compostos. Em +1,20 V, PAR e PRO foram oxidados sem a interferência da CAF. Neste pulso de potencial, a corrente de oxidação da PRO pode ser obtida se a corrente proveniente da oxidação do PAR for previamente subtraída. No pulso de potencial de $+1,60 \mathrm{~V}$, os três compostos foram oxidados e a corrente oxidação da CAF somente pode ser obtida se as correntes de oxidação provenientes do PAR e PRO forem previamente subtraídas. A aplicação do potencial de $0,0 \mathrm{~V} / 100 \mathrm{~ms}$ foi necessária para evitar ou diminuir a contaminação/passivação da superfície do eletrodo de BDD durante os experimentos e obtenção de melhor repetitividade nos resultados.

Adicionalmente, os resultados apresentados na Figura 2 também mostram que a magnitude da corrente de oxidação do PAR em +0,90 $\mathrm{V}(21,6 \mu \mathrm{A})$, em $+1,20 \mathrm{~V}(27,0 \mu \mathrm{A})$ e em $+1,60 \mathrm{~V}(27,3 \mu \mathrm{A})$ e da PRO em +1,20 V $(8,39 \mu \mathrm{A})$ e em +1,60 V $(11,4 \mu \mathrm{A})$ não são iguais. Este fenômeno impede o uso da subtração simples das correntes detectadas nestes pulsos de potenciais para ter acesso às correntes provenientes da oxidação da PRO ( $\left.i_{\text {PRO }}=i_{+1,20 \mathrm{~V}}-i_{+0,90 \mathrm{~V}}\right)$ e da CAF $\left(i_{\text {CAF }}=i_{+1,60 \mathrm{~V}}-i_{+1,20 \mathrm{~V}}-i_{+0,90 \mathrm{~V}}\right)$. Este problema pode ser facilmente contornado por meio da utilização de fatores de correção (FCs), conforme demonstrado anteriormente. ${ }^{24,25,27} \mathrm{Os} \mathrm{FCs} \mathrm{foram} \mathrm{obtidos} \mathrm{pela}$ injeção de uma solução contendo apenas PAR $\left(\mathrm{FC}_{1}\right.$ e $\left.\mathrm{FC}_{2}\right)$ e apenas $\mathrm{PRO}\left(\mathrm{FC}_{3}\right)$ no sistema BIA-MPA e através das seguintes equações:

$$
\begin{aligned}
& F C_{1}=\mathrm{i}_{\text {PAR em }+1,2 \mathrm{v}} / \mathrm{i}_{\text {PAR em }+0,9 \mathrm{v}} \\
& F C_{2}=\mathrm{i}_{\text {PAR em }+1,6 \mathrm{v}} / \mathrm{i}_{\text {PAR em }+0,9 \mathrm{~V}} \\
& F C_{3}=\mathrm{i}_{\text {PRO em }+1,6 \mathrm{~V} /} i_{\text {PRO em }+1,2 \mathrm{~V}}
\end{aligned}
$$

Assim, após a injeção de uma solução contendo simultaneamente PAR, PRO e CAF, a corrente de oxidação proveniente apenas da oxidação da PRO em $+1,20 \mathrm{~V}$ e da oxidação da CAF em $+1,60 \mathrm{~V}$ pode ser calculada por meio das equações (4) e (5).

$$
\begin{gathered}
\mathrm{i}_{\text {PRO }}=\mathrm{i}_{+1,20 \mathrm{~V}}-\left(F C_{l} \times \mathrm{i}_{+0,90 \mathrm{~V}}\right) \\
\mathrm{i}_{\mathrm{CAF}}=\mathrm{i}_{+1,60 \mathrm{~V}}-\left(F C_{2} \times \mathrm{i}_{+0,90 \mathrm{~V}}\right)-\left(F C_{3} \times \mathrm{i}_{\mathrm{PRO}}\right)
\end{gathered}
$$

De forma similar às curvas de calibração, os valores dos FCs devem ser obtidos diariamente antes das análises, uma vez que pequenas variações nos valores dos FCs podem ocorrer em análises realizadas em dias diferentes. Além disso, na definição dos intervalos de concentração a serem utilizados nas curvas de calibração, além de considerar os coeficientes de correlação ( $\mathrm{R} \geq 0,99)$, os FCs também devem apresentar valores constantes. O PAR apresentou valores de FCs constantes $\left(\mathrm{FC}_{1}\right.$ $\left.=1,25 \pm 0,03 ; \mathrm{FC}_{2}=1,26 \pm 0,02\right)$ e boa linearidade $(\mathrm{R} \geq 0,998)$ no intervalo de concentração entre 10 e $85 \mathrm{mg} \mathrm{L}^{-1}$. A PRO apresentou um valor de $\mathrm{FC}$ constante $\left(\mathrm{FC}_{3}=1,29 \pm 0,03\right)$ e linearidade adequada $(\mathrm{R}$ $\geq 0,998$ ) no intervalo de concentração entre 5 e $50 \mathrm{mg} \mathrm{L}^{-1}$.

Na etapa seguinte deste estudo, os parâmetros relacionados com o sistema BIA (volume de injeção, velocidade de injeção e velocidade de agitação da solução no interior da célula) foram estudados e otimizados com o objetivo de obter o melhor desempenho com o sistema. $\mathrm{O}$ volume de injeção e a velocidade de injeção otimizados foram $150 \mu \mathrm{L}$ e $100 \mu \mathrm{L} \mathrm{s}^{-1}$ (melhor sensibilidade e/ou repetividade). A influência da agitação no sistema BIA-MPA foi estudada e foi observado que com o uso de agitação da solução no interior da célula, os picos transientes retornam um pouco mais rapidamente para a linha base, o que causa aumento da frequência analítica do método. Outra vantagem do uso da agitação é que os três compostos e seus respectivos produtos de oxidação são removidos mais rapidamente da superfície do eletrodo de trabalho, reduzindo assim, a possibilidade de contaminação do eletrodo de BDD. A velocidade de agitação otimizada neste trabalho foi de $1400 \mathrm{rpm}$.

A Figura 3 mostra os amperogramas obtidos em três pulsos de potenciais distintos $(+0,9 \mathrm{~V} / 50 \mathrm{~ms},+1,2 \mathrm{~V} / 50 \mathrm{~ms}$ e $+1,6 \mathrm{~V} / 50 \mathrm{~ms})$ para injeções sucessivas de soluções $(n=10)$ contendo as seguintes concentrações: (a) $4+12+20 \mathrm{mg} \mathrm{L}^{-1}$ e (b) $12+36+60 \mathrm{mg} \mathrm{L}^{-1} \mathrm{de}$ $\mathrm{CAF}+\mathrm{PRO}+\mathrm{PAR}$, respectivamente.

Neste estudo, o DPR calculado para o PAR em $+0,9 \mathrm{~V}$ foi de $1,2 \%$ (a) e $1,1 \%$ (b); para a PRO em $+1,2 \mathrm{~V}$ foi de $3,0 \%$ (a) e $2,6 \%$ (b) e para a CAF em $+1,6 \mathrm{~V}$ foi de $3,1 \%$ (a) e 3,9\% (b). Esses resultados indicaram que o método apresentou boa estabilidade $\mathrm{e}$ não houve efeito de memória entre injeções alternadas de soluções contendo concentrações bem diferentes. Entretanto, esses resultados somente foram obtidos quando o pulso de potencial de $0,0 \mathrm{~V} / 100$ ms foi incluído na sequência de pulsos de potencial (Figura 2A) aplicado ao eletrodo de BDD. A aplicação deste pulso de potencial provavelmente foi responsável pela diminuição da contaminação do eletrodo de trabalho. 


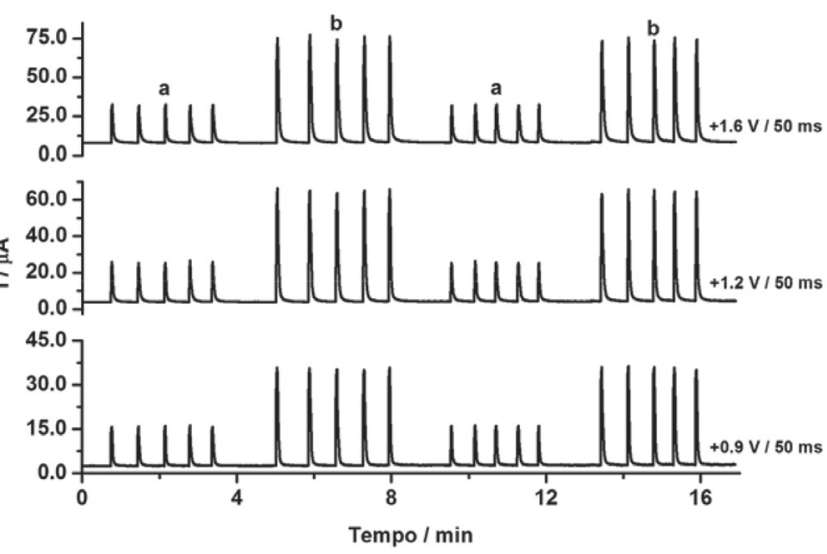

Figura 3. Estudo de repetibilidade. Amperogramas obtidos em três pulsos de potenciais distintos para injeções sucessivas de soluções contendo: (a) PAR $+P R O+C A F\left(20 m g L^{-1}+12 m g L^{-1}+4 m g L^{-1}\right) e(b) P A R+P R O+C A F$ (60 $\mathrm{mg} \mathrm{L}^{-1}+36 \mathrm{mg} \mathrm{L}^{-1}+12 \mathrm{mg} \mathrm{L}^{-1}$ ). Volume de injeção: $150 \mu \mathrm{L}$; velocidade de injeção: $100 \mu \mathrm{L} \mathrm{s} \mathrm{L}^{-1}$; velocidade de agitação: $1400 \mathrm{rpm}$

A Figura 4A apresenta os amperogramas obtidos em três pulsos de potenciais com a injeção em triplicata de soluções contendo somente $20 \mathrm{mg} \mathrm{L}^{-1}$ de PAR (usada para o cálculo do $\mathrm{FC}_{1}$ e $\mathrm{FC}_{2}$ ), somente $12 \mathrm{mg} \mathrm{L}^{-1}$ de $\mathrm{PRO}$ (usada para o cálculo do $\mathrm{FC}_{3}$ ), cinco soluções padrão contendo concentrações crescentes de CAF (a-e: 2 - $\left.10 \mathrm{mg} \mathrm{L}^{-1}\right)$, PRO $\left(\mathrm{a}-\mathrm{e}: 6\right.$ - $\left.30 \mathrm{mg} \mathrm{L}^{-1}\right)$ e PAR $\left(\mathrm{a}-\mathrm{e}: 10\right.$ - $\left.50 \mathrm{mg} \mathrm{L}^{-1}\right)$ e duas amostras $(f-g)$ que foram preparadas adequadamente pela diluição em eletrólito suporte antes da injeção no sistema BIA-MPA. As curvas de calibração preparadas para os três compostos foram construídas levando-se em consideração a faixa de concentração na qual os FCs são relativamente constantes e a proporção existente entre os compostos em amostras farmacêuticas. As respectivas curvas de calibração também são apresentadas (B, C e D).

As curvas de calibração obtidas a partir de dados da Figura 4A para PAR, PRO e CAF apresentaram linearidade satisfatória nas faixas de concentrações estudadas $(r \geq 0,998)$. A Tabela 1 mostra as características analíticas do método proposto.

Tabela 1. Características do método proposto para determinação simultânea de PAR, PRO e CAF

\begin{tabular}{lccc}
\hline Características & PAR & PRO & CAF \\
\hline Faixa linear $\left(\mathrm{mg} \mathrm{L}^{-1}\right)$ & $10-85$ & $5-50$ & $2-16$ \\
$\mathrm{r}$ & 0,998 & 0,999 & 0,998 \\
Slope $\left(\mu \mathrm{A} \mathrm{mg}^{-1} \mathrm{~L}\right)$ & 0,6920 & 0,3665 & 1,2482 \\
Intercepto $(\mu \mathrm{A})$ & 0,0051 & $-0,0013$ & $-0,0098$ \\
$\mathrm{LD}\left(\mathrm{mg} \mathrm{L}^{-1}\right)$ & 0,2 & 0,3 & 0,1 \\
$\mathrm{LQ}\left(\mathrm{mg} \mathrm{L}^{-1}\right)$ & 0,7 & 1,0 & 0,4 \\
FA $\left(\mathrm{h}^{-1}\right)$ & 75 & 75 & 75 \\
DPR intra-dias $(\mathrm{n}=10)$ & $1,2 \%$ & $3,0 \%$ & $3,9 \%$ \\
DPR inter-dias $(\mathrm{n}=3)$ & $4,3 \%$ & $6,1 \%$ & $6,4 \%$ \\
\hline
\end{tabular}

$\mathrm{LD}=$ limite de detecção; $\mathrm{LQ}=$ limite de quantificação; $\mathrm{FA}=$ frequência analítica.

O desempenho do sistema BIA-MPA foi verificado na análise de duas amostras farmacêuticas contendo simultaneamente PAR, PRO e CAF e os resultados obtidos foram comparados com os obtidos por HPLC. A Tabela 2 mostra os resultados obtidos na análise das amostras por ambos os métodos.

O teste $\mathrm{t}$ de Student para amostras pareadas foi usado para comparar os valores obtidos por HPLC com os obtidos por BIA-MPA. A

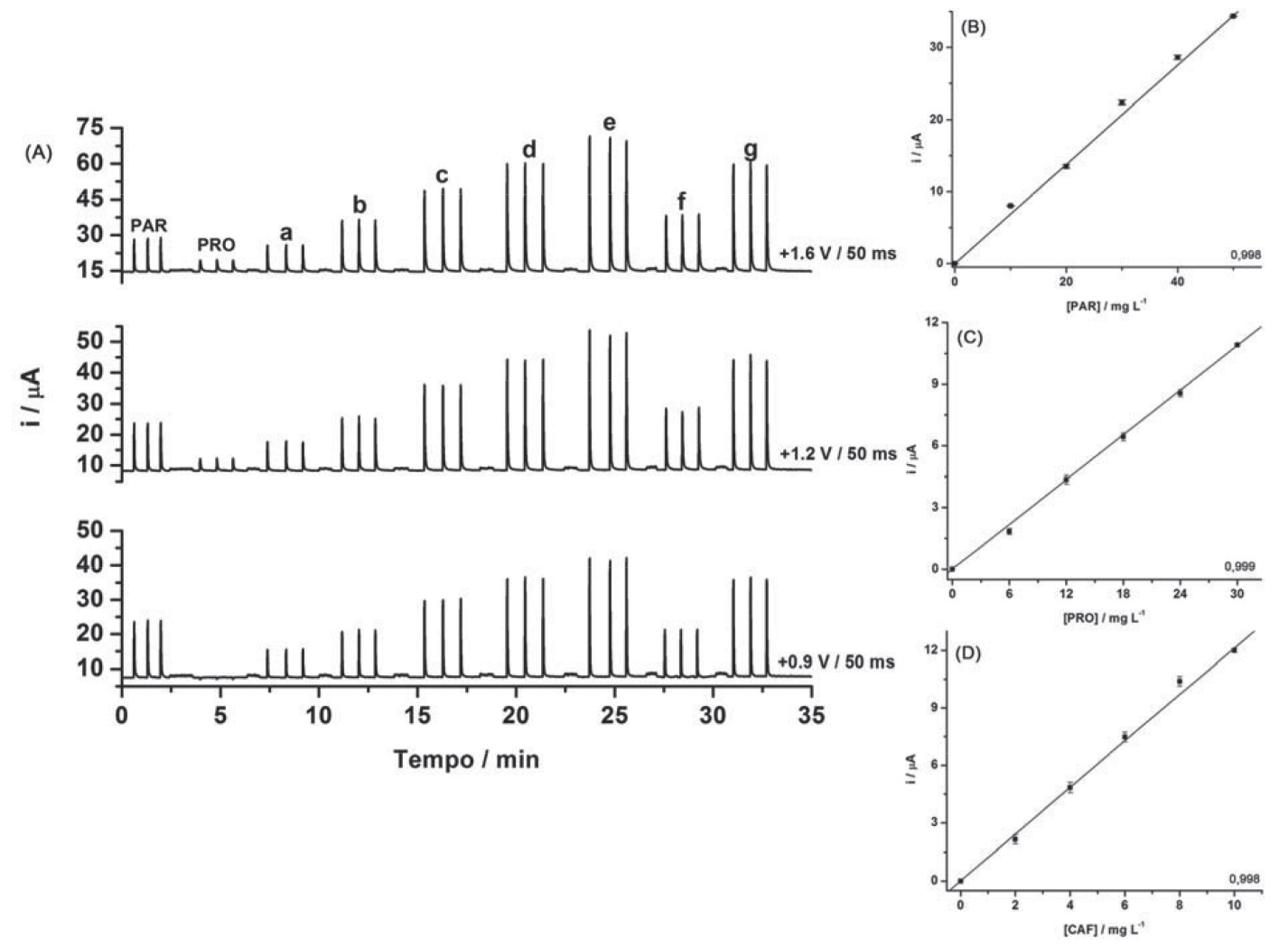

Figura 4. (A) Amperogramas obtidos com o sistema BIA-MPA em três pulsos de potenciais distintos para injeções de soluções contendo somente PAR 20 mg $L^{-1}$, somente PRO $12 \mathrm{mg} L^{l}$, cinco soluções padrão contendo concentrações crescentes de CAF (a-e: $\left.2-10 \mathrm{mg} L^{-1}\right)$, PRO $\left(a-e: 6-30 \mathrm{mg} L^{-1}\right)$ e PAR (a-e: 10

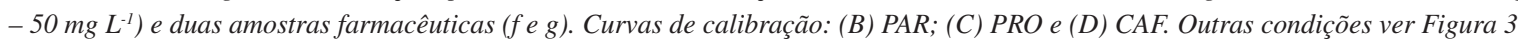


Tabela 2. Comparação entre os resultados obtidos por BIA-MPA e HPLC

\begin{tabular}{|c|c|c|c|c|c|c|}
\hline \multirow[t]{2}{*}{ Amostras } & \multicolumn{2}{|c|}{ Valor da bula (mg/drágea) } & \multirow{2}{*}{$\begin{array}{c}\begin{array}{c}\text { BIA-MPA } \\
\text { (mg/drágea) }\end{array} \\
251 \pm 3\end{array}$} & \multirow{2}{*}{$\begin{array}{c}\begin{array}{c}\text { HPLC } \\
\text { (mg/drágea) }\end{array} \\
258 \pm 5\end{array}$} & \multirow{2}{*}{$\begin{array}{c}\mathrm{E}^{1} \\
(\%) \\
-2,7\end{array}$} & \multirow{2}{*}{$\begin{array}{c}\mathrm{E}^{2} \\
(\%) \\
+0,4\end{array}$} \\
\hline & PAR & 250 & & & & \\
\hline \multirow[t]{3}{*}{1} & PRO & 150 & $154 \pm 3$ & $152 \pm 5$ & $+1,3$ & $+2,7$ \\
\hline & CAF & 50 & $52 \pm 4$ & $53 \pm 1$ & $-1,9$ & $+4,0$ \\
\hline & PAR & 250 & $249 \pm 3$ & $257 \pm 5$ & $-3,1$ & $-0,4$ \\
\hline \multirow[t]{2}{*}{2} & PRO & 150 & $149 \pm 4$ & $159 \pm 5$ & $-6,3$ & $-0,7$ \\
\hline & $\mathrm{CAF}$ & 50 & $49 \pm 4$ & $52 \pm 1$ & $-5,8$ & $-2,0$ \\
\hline
\end{tabular}

$\mathrm{E}^{1}: 100 \times(\mathrm{BIA}-\mathrm{HPLC}) / \mathrm{HPLC} ; \mathrm{E}^{2}: 100 \times(\mathrm{BIA}-$ Valor da bula $) /$ Valor da bula.

partir dos resultados apresentados na Tabela 2 pode-se afirmar que os resultados obtidos pelo método proposto foram similares aos obtidos por HPLC a um nível de confiança de $95 \%$, onde os valores de $\mathrm{t}$ calculados foram menores que o valor crítico teórico $(2,78, \mathrm{n}=3)$.

Os resultados obtidos com o método BIA-MPA também foram comparados aos obtidos com outros métodos da literatura ${ }^{2,9,13,14,17-19,21}$ que também possibilitam a determinação simultânea de PAR, PRO e CAF (Tabela 3). Como pode ser observado, alguns trabalhos publicados anteriormente apresentam limites de detecção mais baixos, porém, para a determinação de princípios ativos em formulações farmacêuticas, baixos limites de detecção geralmente não são necessários. Como vantagens do método aqui proposto podem ser citados a frequência analítica muito superior, preparo de amostras simplificado (simples pulverização, dissolução e diluição) e geração mínima de resíduos por análise (método analítico verde).

\section{CONCLUSÃO}

O sistema BIA-MPA foi utilizado com sucesso na determinação rápida e simultânea de PAR, PRO e CAF em amostras farmacêuticas.

Tabela 3. Comparação entre as características analíticas do método proposto e métodos da literatura que possibilitam a determinação simultânea de PAR, PRO e CAF.

\begin{tabular}{|c|c|c|c|c|c|c|c|c|}
\hline Método & Composto & $\begin{array}{l}\text { Faixa linear } \\
\left(\mathrm{mg} \mathrm{L}^{-1}\right)\end{array}$ & $\mathrm{r}$ & $\begin{array}{c}\mathrm{LD} \\
\left(\mathrm{mg} \mathrm{L}^{-1}\right)\end{array}$ & $\begin{array}{c}\mathrm{LQ} \\
\left(\mathrm{mg} \mathrm{L}^{-1}\right)\end{array}$ & $\begin{array}{c}\text { DPR } \\
(\%)\end{array}$ & $\begin{array}{l}\text { FA } \\
\left(h^{-1}\right)\end{array}$ & Ref \\
\hline \multirow{3}{*}{ BIA-MPA } & PAR & $10-85$ & 0,998 & 0,2 & 0,7 & 1,2 & 75 & \\
\hline & PRO & $5-50$ & 0,999 & 0,3 & 1,0 & 3,0 & 75 & $*$ \\
\hline & $\mathrm{CAF}$ & $2-16$ & 0,998 & 0,1 & 0,4 & 3,9 & 75 & \\
\hline \multirow{3}{*}{ BIA-SWV } & PAR & $2-50$ & 0,997 & 0,3 & 1,0 & 1,9 & 80 & \\
\hline & PRO & $2-50$ & 0,998 & 0,2 & 0,7 & 1,5 & 80 & 21 \\
\hline & $\mathrm{CAF}$ & $2-50$ & 0,997 & 0,3 & 1,0 & 3,1 & 80 & \\
\hline \multirow{3}{*}{ SWV } & PAR & $1-50$ & 0,999 & 0,005 & 0,02 & 1,0 & 20 & \\
\hline & PRO & $1-50$ & 0,999 & 0,009 & 0,03 & 1,0 & 20 & 21 \\
\hline & $\mathrm{CAF}$ & $1-40$ & 0,999 & 0,006 & 0,02 & 4,1 & 20 & \\
\hline \multirow{3}{*}{ HPLC-UV } & PAR & $1-10$ & - & 0,015 & - & 3,8 & 7 & \\
\hline & PRO & $1-10$ & - & 0,005 & - & 4,2 & 7 & 13 \\
\hline & $\mathrm{CAF}$ & $1-10$ & - & 0,010 & - & 4,2 & 7 & \\
\hline \multirow{3}{*}{ HPLC-UV } & PAR & $3-90$ & 0,999 & 0,3 & 0,8 & 3,0 & 5 & \\
\hline & PRO & $2-45$ & 0,999 & 0,3 & 1,0 & 3,0 & 5 & 9 \\
\hline & CAF & $1-15$ & 0,999 & 0,04 & 0,13 & 3,0 & 5 & \\
\hline \multirow{3}{*}{ HPLC-UV } & PAR & $6-420$ & 0,995 & 2,2 & 4,6 & 1,3 & 5 & \\
\hline & PRO & $1-240$ & 0,993 & 0,1 & 0,12 & 1,7 & 5 & 14 \\
\hline & $\mathrm{CAF}$ & $1-80$ & 0,994 & 0,4 & 0,62 & 1,7 & 5 & \\
\hline \multirow{3}{*}{ HPLC-UV } & PAR & $43-128$ & 0,999 & 0,04 & 0,125 & 0,07 & 3 & \\
\hline & PRO & $25-75$ & 0,999 & 0,19 & 0,776 & 0,05 & 3 & 2 \\
\hline & $\mathrm{CAF}$ & $9-25$ & 0,999 & 0,07 & 0,295 & 0,03 & 3 & \\
\hline \multirow{3}{*}{ SP-UV/Vis } & PAR & $5-25$ & 0,999 & - & - & - & - & \\
\hline & PRO & $5-25$ & 0,999 & - & - & - & - & 17 \\
\hline & $\mathrm{CAF}$ & $1-5$ & 0,999 & - & - & - & - & \\
\hline \multirow{3}{*}{ SP-UV/FIA } & PAR & $25-350$ & 0,999 & 7,5 & 25 & 3,5 & 11 & \\
\hline & PRO & $15-150$ & 0,999 & 1,9 & 6,2 & 3,8 & 11 & 18 \\
\hline & $\mathrm{CAF}$ & $5-75$ & 0,999 & 0,7 & 2,2 & 3,7 & 11 & \\
\hline \multirow{3}{*}{ MEKC } & PAR & $2-200$ & 0,999 & 0,6 & 2 & 1,5 & 7 & \\
\hline & PRO & $3-200$ & 0,999 & 0,8 & 3 & 1,7 & 7 & 19 \\
\hline & CAF & $2-200$ & 0,999 & 0,6 & 2 & 1,7 & 7 & \\
\hline
\end{tabular}

*Este trabalho; LD: limite de deteç̧ão; LQ: limite de quantificação; FA: frequência analítica; BIA-SWV: Análise por injeção em batelada com detecção por voltametria de onda quadrada; SP: espectrofotometria; MEKC: cromatografia eletrocinética micelar. 
O método apresenta vantagens como: baixo custo relacionado com a aquisição e manutenção de equipamentos, baixo consumo de amostras de reagentes, preparo de amostras simplificado (apenas pulverização, dissolução e diluição) e alta frequência analítica ( 75 injeções $\mathrm{h}^{-1}$ ). Além disto, os resultados obtidos pelo método BIA-MPA são similares aos obtidos por HPLC, a um nível de confiança de $95 \%$.

\section{AGRADECIMENTOS}

Os autores agradecem o suporte financeiro da CAPES (PRO FORENSES - Processo número 23038.007073/2014-12), do CNPQ (Processo número 307333/2014-0) e da FAPEMIG (CEX - APQ-02118-15).

\section{REFERÊNCIAS}

1. Mazzarino, M.; Braganò, M. C.; Donati, F.; de la Torre, X.; Botrè, F.; Anal. Chim. Acta 2010, 657, 60 .

2. Soponar, F.; Staniloae, D.; Moise, G.; Szaniszlo, B.; David, V.; Rev. Roum. Chim. 2013, 58, 433.

3. Kiersch, T. A.; Minić, M. R.; Curr. Med. Res. Opin. 2002, 18, 18.

4. Chang, Z.; Gao, N.; Li, Y.; He, X.; Anal. Methods 2012, 4, 4037.

5. Lourenção, B. C.; Medeiros, R. A.; Rocha-Filho, R. C.; Mazo, L. H.; Fatibello-Filho, O; Talanta 2009, 78, 748.

6. Yin, H.; Shang, K.; Meng, X.; Ai, S.; Microchim. Acta 2011, 175, 39.

7. Bosch, M. E.; Sánchez, A. J. R.; Rojas, F. S.; Ojeda, C. B.; J. Pharm. Biomed. Anal. 2006, 42, 291.

8. Prabakar, S. J. R.; Narayanan, S. S.; Talanta 2007, 72, 1818.

9. Delvadiya, K.; Kabra, P.; Kimbahune, R.; Patel, N.; Nargund, L.; Indian J. Pharm. Educ. Res. 2014, 47, 65.

10. Švorc, L.; Tomčík, P.; Svítková, J.; Rievaj, M.; Bustin, D.; Food Chem. 2012, 135, 1198.

11. Burdan, F.; Hum. Exp. Toxicol. 2004, 23, 235.

12. Hałka-Grysińska, A.; Ślązak, P.; Zaręba, G.; Markowski, W.; KlimekTurek, A.; Dzido, T. H.; Anal. Methods 2012, 4, 973.

13. Avramova, J.; J. Pharm. Biomed. Anal. 1989, 7, 1221.

14. Issa, Y. M.; Hassouna, M. E. M.; Zayed, A. G.; J. Liq. Chromatogr. Relat. Technol. 2012, 35, 2148.

15. Dinç, E.; Kökdil, G.; Onur, F.; J. Pharm. Biomed. Anal. 2001, 26, 769.

16. Dumanovic, D.; Markovic, D.; Muk, A.; Talanta 1989, 36, 931.

17. Özgür, M. Ü.; Alpdoğan, G.; Aşçi, B.; Monatsh. Chem. 2002, 133, 219.

18. Vidal, A. D.; Barrales, P. O.; Díaz, A. M.; Microchim. Acta 2003, 141, 157.

19. Emre, D.; Özaltin, N.; J. Chromatogr. B 2007, 847, 126.
20. Hałka-Grysińska, A.; Ślązak, P.; Zaręba, G.; Markowski, W.; KlimekTurek, A.; Dzido, T.; H.; Anal. Methods 2012, 4, 973.

21. Silva, W. P.; Silva, L. A. J.; França, C. H.; Sousa, R. M. F.; Muñoz, R. A. A.; Richter, E. M.; Electroanalysis 2017, 29, 1860.

22. Pereira, P. F.; da Silva, W. P.; Muñoz, R. A. A.; Richter, E. M.; Quim. Nova 2015, 38, 663.

23. Pereira, P. F.; da Silva, W. P.; Marra, M. C.; Muñoz, R. A. A.; Richter, E. M.; Anal. Methods 2016, 44, 7959.

24. Freitas, J. M.; da Costa Oliveira, T.; Gimenes, D. T.; Munoz, R. A. A.; Richter, E. M.; Talanta 2016, 146, 670.

25. Pereira, P. F.; da Silva, W. P.; Muñoz, R. A. A.; Richter, E. M.; J. Electroanal. Chem. 2016, 766, 87.

26. Quintino, M.; S.; M.; Angnes, L.; Electroanalysis 2004, 16, 513.

27. Wang, J.; Taha, Z.; Anal. Chem. 1991, 63, 1053.

28. Tormin, T. F.; Cunha, R. R.; da Silva, R. A. B.; Munoz, R. A. A.; Richter, E. M.; Sens. Actuators, B 2014, 202, 93.

29. Pedrotti, J. J.; Angnes, L. L.; Gutz, I. G. R.; Electroanalysis 1996, 8, 673.

30. Salazar-Banda, G. R.; Andrade, L. S.; Nascente, P. A. P.; Pizani, P. S.; Rocha-Filho, R. C.; Avaca, L. A.; Electrochim. Acta 2006, 51, 4612.

31. Terashima, C.; Rao, T. N.; Sarada, B. V.; Kubota, Y.; Fujishima, A.; Anal. Chem. 2003, 75, 1564.

32. Tormin, T. F.; Gimenes, D. T.; Richter, E. M.; Munoz, R. A. A.; Talanta 2011, 85, 1274.

33. Pereira, P. F.; Marra, M. C.; Munoz, R. A A.; Richter, E. M.; Talanta 2012, 90, 99.

34. Quintino, M. S. M.; Angnes, L.; Electroanalysis 2004, 16, 513.

35. Richter, E. M.; Tormin, T. F.; Cunha, R. R.; Silva, W. P.; Pérez-Junquera, A.; Fanjul-Bolado, P.; Hernández-Santos, D.; Electroanalysis 2016, 28, 1856.

36. Oliveira, T. C.; Freitas, J. M.; Munoz, R. A. A.; Richter, E. M.; Talanta 2016, 152, 308.

37. Caramit, R. P.; Lucca, B. G.; Ferreira. V. S.; Munoz, R. A. A.; Richter, E. M.; da Silva, R. A. B.; Electroanalysis 2015, 27, 271.

38. https://www.youtube.com/watch?v=oe0G2RA_t4o, acessado em setembro 2017.

39. https://www.youtube.com/watch? $v=j 2 X q L r \_C y X k$, acessado em setembro 2017.

40. dos Santos, W. T. P.; De Almeida, E.; G.; N.; Ferreira, H. E. A.; Gimenes, D. T.; Richter, E. M.; Electroanalysis 2008, 20, 1878.

41. Wangfuengkanagul, N.; Chailapakul, O.; J. Pharm. Biomed. Anal. 2002, 28,841 .

42. Spãtaru, N.; Sarada, B. V.; Tryk, D. A.; Fujishima, A.; Electroanalysis 2002, 14,721 